\title{
Editorials
}

\section{Optimizing transfusion practice}

\author{
Nadine Shehata MD MSC FRCPC, ${ }^{*}$ C. David Mazer MD FRCPC $\dagger$
}

$\mathrm{T}$ RANSFUSION therapy has undergone several changes over the years with supply challenges and the realization that blood transfusions are not innocuous. Blood conservation strategies have taken the forefront in research communities. Hematinic agents have been advocated as a means of increasing the hemoglobin concentration to counterbalance blood loss. Reducing preoperative exposure to anticoagulants has been debated to reduce the risk of perioperative bleeding. Alternatives to allogeneic blood such as autologous blood (i.e., preoperative autologous donation, acute normovolemic hemodilution, red cell salvage) have gained some acceptance in their role in reducing exposure to allogeneic blood.

In addition to these strategies, the methods by which we assess the indication for transfusion have come into question. In the past, it was widely accepted that a decrease in hemoglobin concentration was associated with a decreased oxygen carrying capacity and that allogeneic transfusions increased tissue oxygenation. Now the benefit of transfusions has come into question, as efficacy has never been determined., ${ }^{1,2}$ Reports that address transfusion therapy have tended to focus on the risks associated with transfusion, ${ }^{3,4}$ and not the benefit of transfusion. Consequently, we have not defined the optimum circumstances for transfusion therapy.

How do we best determine when a transfusion is indicated? We can attempt to provide evidence that impaired tissue oxygenation improves with a transfusion, but this has proven to be a difficult task. Ideally, the intracellular partial pressure of oxygen should be directly measured to determine tissue oxygenation, ${ }^{5}$ but this cannot be done in clinical settings. In addition, there is significant interpatient variability in the critical point of oxygen delivery. Thus, the terminol- ogy associated with transfusion practice should be re-evaluated. Words such as "need" for a transfusion or transfusion "requirements" should be replaced with phrases such as "received a transfusion" or "transfusions administered" since the data to indicate when a transfusion is actually "necessary" is lacking.

How do we then optimize transfusion practice? There are several ways. We can explore alternate surrogate biomarkers that assess adequate tissue oxygenation. We can continue to investigate various alternatives to blood components to limit exposure to allogeneic blood. We can better educate caregivers and patients regarding the risks of transfusions. We can disseminate information regarding optimum practices through practice guidelines. We can also try to understand physicians' rationale for transfusions and prioritize research to focus on common triggers for transfusion.

One preliminary way to understand the rationale for any decision is to survey a population using simulated patients to assess medical decision-making. The benefit of the survey technique is that it facilitates information gathering from a large population. In addition, surveys permit investigators to study attitudes regarding transfusions, and the associations between patient or physician factors and transfusion decisions. Ultimately results from surveys can be used to explore the associations between variables (i.e., patient and physicians factors) and outcomes (i.e., hemoglobin thresholds).

In this issue of the Journal, Turgeon et al. have pursued this end by surveying Canadian anesthesiologists to assess attitudes towards transfusions of red blood cells. ${ }^{6}$ The survey included three scenario-based questions to determine hemoglobin thresholds during general, orthopedic and vascular surgery, and to elicit information on the utilization of blood conserva-

From the Department of Medicine and Canadian Blood Services, ${ }^{*}$ and the Department of Anesthesia, $†$ St. Michael's Hospital, University of Toronto, Toronto, Ontario, Canada.

Address correspondence to: Dr. C. David Mazer, Department of Anesthesia, St. Michael's Hospital, 30 Bond Street, Toronto,

ON M5B 1W8, Canada. Phone: 416-864-5825; Fax: 416-864-6014; E-mail: mazerd@smh.toronto.on.ca 
tion techniques, and the importance of information resources that affect transfusion practices. Physicians were asked to select the lowest hemoglobin threshold they would select for each patient, and the scenarios were then modified by sex, the presence of coronary artery disease, age, preoperative chronic anemia, availability of autologous blood, and increased perioperative bleeding.

The hemoglobin thresholds selected for transfusion varied with patient risk factors. Overall, higher hemoglobin thresholds were selected for the vascular surgery case scenarios compared to the orthopedic and general surgery scenario. When patient-specific factors such as the presence of coronary artery disease and age were introduced, clinically significant changes in hemoglobin thresholds emerged. In the presence of coronary artery disease, $20 \%$ to $50 \%$ of respondents selected a hemoglobin threshold of $100 \mathrm{~g} \cdot \mathrm{L}^{-1}$ or more compared to less than $10 \%$ of physicians for the baseline case scenario in the absence of coronary artery disease. The age of the simulated patient also resulted in clinically significant changes in the hemoglobin thresholds selected. As age increased from $20 \mathrm{yr}$ to $60 \mathrm{yr}$, a significantly lower proportion of respondents selected a hemoglobin threshold of $70 \mathrm{~g} \cdot \mathrm{L}^{-1}$ or less (92\% compared to $52 \%$ respectively for the general surgery case scenario; $P<0.001$ ).

These results coincide with the trial of Transfusion Triggers in Critical Care (TRICC), ${ }^{7}$ the most recent survey of the American Society of Anesthesiologists $(\mathrm{ASA})^{8}$ and the recently updated guidelines by the ASA's Task Force. ${ }^{9}$ The TRICC $^{7}$ trial demonstrated that transfusing critically ill patients at a hemoglobin concentration of $70 \mathrm{~g} \cdot \mathrm{L}^{-1}$ did not result in an increased morbidity and mortality compared with a hemoglobin threshold of less than $100 \mathrm{~g} \cdot \mathrm{L}^{-1}$. The survey and guidelines by the ASA suggest that perioperative hemoglobin thresholds have decreased over the years. The proportion of anesthesiologists who indicated they would require patients to have a hemoglobin concentration of at least $100 \mathrm{~g} \cdot \mathrm{L}^{-1}$ had decreased from $65 \%$ in an earlier survey ${ }^{10}$ to $9 \%$ in $2002 .{ }^{8}$ In the study by Turgeon et al., less than $10 \%$ of physicians selected a hemoglobin threshold of $100 \mathrm{~g} \cdot \mathrm{L}^{-1}$ for transfusion. ${ }^{6}$ The ASA practice guidelines also recommend that red cells are usually unnecessary when the hemoglobin level is more than $100 \mathrm{~g} \cdot \mathrm{L}^{-1}$.

Interestingly, the study by Turgeon et al. did not demonstrate that blood conservation techniques are universally utilized. Seventy-five percent of anesthesiologists indicated they never or rarely use erythropoietin, $53 \%$ indicated they used iron therapy frequently, and only $31 \%$ indicated they used autologous blood frequently. Physicians who selected transfusion thresholds of less than $70 \mathrm{~g} \cdot \mathrm{L}^{-1}$ were more likely to use blood conservation techniques. Contrary to this finding, the ASA survey reported that the use of apparatus for intraoperative salvage and reinfusion of blood increased from $39 \%$ in 1981 to $95 \%$ in $2002 .{ }^{8}$ The ASA guidelines also advocate the use of blood conservation strategies for the prevention or reduction of allogeneic transfusion requirements. ${ }^{9}$

The primary limitation to the study by Turgeon et al. is related to sampling. Only members of the Canadian Anesthesiologists' Society were surveyed and the response rate was $47 \%$. The small sample size presents the possibility that the results might not be indicative of the entire population i.e., including the remaining $53 \%$ of the population might have altered the results reported.

Nonetheless, the effect of myocardial ischemia and increasing patient age on hemoglobin thresholds represent two areas of controversy in transfusion medicine. Although the ASA survey also identified age and the medical condition of the patient as factors affecting transfusion decisions ${ }^{8}$ and the practice guidelines support the selection of hemoglobin thresholds based on risk factors of inadequate oxygenation, ${ }^{9}$ the optimum hemoglobin thresholds based on these factors have still not been established.

Transfusions in patients with myocardial ischemia have received much attention because of two reports with somewhat disparate results on the optimum hemoglobin thresholds in patients with acute coronary syndromes. ${ }^{11,12}$ In a large retrospective database study of Medicare patients, ${ }^{11} \mathrm{Wu}$ et al. suggested that patients with acute myocardial ischemia would benefit from transfusions at a hemoglobin concentration of $100 \mathrm{~g} \cdot \mathrm{L}^{-1}$. A subsequent report suggested that a hemoglobin concentration of $80 \mathrm{~g} \cdot \mathrm{L}^{-1}$ may result in better outcomes, but transfusion at a hemoglobin concentration of $100 \mathrm{~g} \cdot \mathrm{L}^{-1}$ would result in increased mortality. ${ }^{12}$ If, as Turgeon et al. indicated in their conclusion, the increase in the hemoglobin thresholds selected with the presence of myocardial ischemia may have resulted from the influence of the report of Wu et al., ${ }^{11}$ physicians may be experiencing uncertainty as to the optimum transfusion threshold based on the subsequent study. ${ }^{12}$ Future studies are urgently needed to address this issue.

The influence of age on hemoglobin thresholds represents a more contentious matter. The adage that elderly people are less tolerant of anemia is not supported by good scientific evidence. Indeed, small studies have shown that elderly individuals tolerate hemodilution to a hematocrit less than $30 \%{ }^{13}$ In 
addition, the results of the TRICC trial did not demonstrate a difference in mortality for patients over the age of $55 \mathrm{yr}$ who were transfused at a hemoglobin concentration of $70 \mathrm{~g} \cdot \mathrm{L}^{-1}$ compared to a hemoglobin concentration of less than $100 \mathrm{~g} \cdot \mathrm{L}^{-1} \cdot{ }^{7}$ Yet, it is well reported that age is a risk factor for transfusion. ${ }^{14}$ The question of whether age should affect hemoglobin thresholds needs to be answered since with an aging population, transfusions based on age alone may impact red cell utilization.

The road to optimizing transfusion practice requires several steps. Besides investigating alternatives to transfusion, and physiological markers for decreased oxygenation, we need to better understand what factors influence clinical decision-making in transfusion practices, whether they are patient-related factors, institutional policies, and/or current best evidence from peer-reviewed publications. To this end, Turgeon et al. provide us with new information. The next steps are to determine whether transfusion based on the factors identified in this study impact on patient morbidity and mortality.

\section{Une pratique transfu- sionnelle optimale}

La thérapeutique transfusionnelle a changé au cours des années avec les défis des réserves de sang et la prise de conscience que les transfusions sanguines ne sont pas sans danger. Les stratégies de conservation du sang sont au premier plan dans les milieux de la recherche. Les agents hématiniques ont été préconisés pour accroître la concentration d'hémoglobine et contrebalancer ainsi la perte de sang. On a débattu d'une réduction de l'exposition préopératoire aux anticoagulants pour diminuer le risque de saignement périopératoire. L'usage de sang autologue (un don autologue préopératoire, une hémodilution normovolémique aiguë, une récupération des globules rouges) est de plus en plus accepté pour réduire l'exposition au sang allogénique.

Outre ces stratégies, les méthodes pour évaluer les indications de transfusion ont été mises en doute. Dans le passé, il était généralement accepté qu'une baisse de la concentration d'hémoglobine soit associée à une réduction de la capacité de transport d'oxygène et que les transfusions allogéniques augmentaient l'oxygénation tissulaire. Le bénéfice des transfusions est maintenant remis en question, puisque l'efficacité n'a jamais été prouvée. ${ }^{1,2}$ La recherche sur la thérapeutique transfusionnelle a surtout insisté sur les risques, non sur les avantages, associés à la transfusion. ${ }^{3,4}$ Nous n'avons donc pas défini le contexte optimal de la thérapie transfusionnelle.

Comment pouvons-nous le mieux déterminer quand une transfusion est indiquée? Nous pouvons tenter de prouver que l'oxygénation tissulaire altérée s'améliore avec une transfusion, mais cela s'est révélé difficile à faire. Idéalement, la pression intracellulaire partielle en oxygène doit être directement mesurée pour déterminer l'oxygénation tissulaire, ${ }^{5}$ ce qui ne peut se faire en clinique. De plus, il y a une variabilité interpatient significative du point critique d'apport en oxygène. Il faut donc réévaluer la terminologie associée à la pratique transfusionnelle. Des mots comme «il faut» une transfusion ou «exigences» de transfusion doivent être remplacés par «transfusion reçue» ou «transfusions administrées» puisque les données manquent pour indiquer quand une transfusion est effectivement «nécessaire».

Comment rendre la pratique transfusionnelle optimale ? Nous pouvons explorer d'autres biomarqueurs substituts qui évaluent l'oxygénation tissulaire adéquate. Nous pouvons chercher encore diverses sources de remplacement des composants sanguins pour limiter l'exposition au sang allogénique. Nous pouvons mieux informer les soignants et les patients sur les risques des transfusions. Nous pouvons diffuser l'information sur les pratiques optimales au moyen de directives cliniques. Nous pouvons aussi essayer de comprendre pourquoi les médecins administrent des transfusions et donner la priorité à la recherche centrée sur les motifs habituels de transfuser.

Une première façon de comprendre le motif de toute décision est de faire une enquête auprès d'une population en utilisant la simulation de patients pour évaluer la prise de décision médicale. L'enquête facilite la cueillette d'informations provenant d'une grande population. Elle permet aussi aux chercheurs d'étudier les attitudes face aux transfusions et les associations entre les facteurs concernant le patient ou le médecin et les décisions de transfuser. Enfin, les résultats des enquêtes peuvent être utilisés pour explorer les associations entre les variables (les facteurs qui concernent le patient et les médecins) et les résultats (niveaux d'hémoglobine par exemple).

Dans le présent numéro du Journal, Turgeon et coll. ont, dans ce but, enquêté auprès des anesthésiologistes canadiens sur les attitudes face aux transfusions de culots globulaires. ${ }^{6}$ L'enquête comprenait trois questions fondées sur des scénarios pour déter- 
miner les seuils d'hémoglobine pendant la chirurgie générale, orthopédique et vasculaire et pour obtenir de l'information sur l'utilisation de techniques de conservation du sang et sur l'importance des ressources d'information touchant les pratiques de transfusion. On a demandé quel serait le plus bas seuil d'hémoglobine sélectionné pour chaque patient et les scénarios ont été modifiés selon le sexe, la présence de cardiopathie ischémique, l'âge, la présence d'anémie préopératoire chronique, la disponibilité de sang autologue et les saignements périopératoires importants.

Les seuils d'hémoglobine choisis pour la transfusion variaient avec les facteurs de risque pour les patients. En général, des seuils plus élevés ont été choisis pour les scénarios de chirurgie vasculaire comparés aux scénarios de chirurgie orthopédique et générale. Quand des facteurs spécifiques au patient étaient introduits, comme la présence de cardiopathie ischémique et l'âge, le choix des seuils d'hémoglobine a changé de façon significative au plan clinique. En cas de cardiopathie ischémique, de 20 à $50 \%$ des répondants ont choisi un seuil de $100 \mathrm{~g} \cdot \mathrm{L}^{-1}$ ou plus comparé à moins de $10 \%$ de médecins pour le scénario initial en l'absence de cardiopathie ischémique. L'âge du patient simulé a aussi amené à modifier significativement le choix des seuils d'hémoglobine. Avec l'âge qui passe de 20 à 60 ans, une proportion significativement plus faible de répondants a choisi un seuil d'hémoglobine de 70 g. $\mathrm{L}^{-1}$ ou moins $(92 \%$ comparé à $52 \%$ respectivement pour le cas de chirurgie général ; $P<0,001$ ).

Ces résultats coïncident avec l'essai de Transfusion Triggers in Critical Care (TRICC), ${ }^{7}$ la plus récente enquête de l' American Society of Anesthesiologists $(\mathrm{ASA})^{8}$ et les directives récemment mises à jour par l'ASA's Task Force. ${ }^{9}$ L'essai TRICC ${ }^{7}$ a démontré que la transfusion faite chez des patients gravement malades à une concentration d'hémoglobine de 70 g. $\mathrm{L}^{-1}$ n'augmente pas la morbidité et la mortalité comparativement à un seuil d'hémoglobine de moins de $100 \mathrm{~g} \cdot \mathrm{L}^{-1}$. L'enquête et les directives de l'ASA laissent voir que les seuils d'hémoglobine périopératoires ont diminué avec le temps. La proportion d'anesthésiologistes qui ont indiqué qu'ils exigeraient une concentration d'hémoglobine d'au moins 100 $\mathrm{g} \cdot \mathrm{L}^{-1}$ est passée de $65 \%$ dans une enquête antérieure à 9 $\%$ en 2002. ${ }^{8}$ Dans l'étude de Turgeon et coll., moins de $10 \%$ des médecins ont choisi un seuil d'hémoglobine de $100 \mathrm{~g} \cdot \mathrm{L}^{-1}$ pour la transfusion. ${ }^{6}$ Les directives cliniques de l'ASA indiquent aussi que les culots globulaires ne sont habituellement pas nécessaires quand le niveau d'hémoglobine est de plus de $100 \mathrm{~g} \cdot \mathrm{L}^{-1}$. ${ }^{9}$

L'étude de Turgeon et coll. n'a pas démontré que les techniques de conservation du sang sont univer- sellement utilisées. Soixante-quinze pour cent des anesthésiologistes ont indiqué qu'ils n'utilisent jamais ou rarement d'érythropoïétine, $53 \%$ qu'ils ont utilisé fréquemment un traitement martial et seulement $31 \%$ qu'ils ont utilisé fréquemment du sang autologue. Les médecins qui ont choisi des seuils de transfusion de moins de $70 \mathrm{~g} \cdot \mathrm{L}^{-1}$ utilisaient plus les techniques de conservation du sang. Par ailleurs, l'enquête de l'ASA a rapporté que l'usage d'appareil pour la récupération peropératoire et la reperfusion de sang est passé de $39 \%$ en 1981 à $95 \%$ en $2002 .{ }^{8}$ Les directives de l'ASA recommandent aussi l'usage de stratégies de conservation du sang pour la prévention ou la réduction des besoins de transfusion allogénique. ${ }^{9}$

La principale limite de l'étude de Turgeon et coll. est reliée à l'échantillonnage. Seuls les membres de la Société canadienne des anesthésiologistes ont été sondés et le taux de réponse a été de $47 \%$. L'échantillonnage de faible effectif présente la possibilité que le résultat ne soit pas indicatif de la population entière, c'est-àdire que l'inclusion des autres $53 \%$ de la population puisse avoir altéré les résultats publiés.

Néanmoins, l'effet de l'ischémie myocardique et de l'âge du patient sur les seuils d'hémoglobine représente deux champs de controverse en médecine transfusionnelle. Quoique l'enquête de l'ASA ait aussi reconnu l'âge et l'état médical du patient comme des facteurs affectant la décision de transfuser ${ }^{8}$ et que les directives cliniques appuient la sélection de seuils d'hémoglobine fondée sur les facteurs de risque d'oxygénation inadéquate, ${ }^{9}$ les seuils d'hémoglobine optimaux fondés sur ces facteurs n'ont pas encore été établis.

Les transfusions chez les patients qui présentent de l'ischémie myocardique ont reçu plus d'attention à cause de deux articles aux résultats plutôt disparates sur les seuils d'hémoglobine optimaux en présence de syndromes coronariens aigus. ${ }^{11,12}$ Dans une grande étude rétrospective des données sur les patients de Medicare, ${ }^{11} \mathrm{Wu}$ et coll. ont pensé que les patients atteints d'ischémie myocardique pourraient bénéficier de transfusions à une concentration d'hémoglobine de $100 \mathrm{~g} \cdot \mathrm{L}^{-1}$. Un article subséquent a suggéré qu'une concentration d'hémoglobine de $80 \mathrm{~g} \cdot \mathrm{L}^{-1}$ pouvait produire de meilleurs résultats, mais que la transfusion à une concentration de $100 \mathrm{~g} \cdot \mathrm{L}^{-1}$ augmenterait la mortalité. ${ }^{12} \mathrm{Si}$, comme l'ont indiqué Turgeon et coll. dans leur conclusion, l'augmentation des seuils d'hémoglobine sélectionnés en présence d'ischémie myocardique a pu être le résultat de l'influence de l'article de Wu et coll., ${ }^{11}$ les médecins peuvent éprouver de l'incertitude quant au seuil de transfusion optimal fondé sur l'étude subséquente. ${ }^{12} \mathrm{D}$ 'autres études sont instamment demandées pour régler cette question. 
L'influence de l'âge sur les seuils d'hémoglobine représente une plus grande controverse. L'opinion voulant que les gens âgés soient moins tolérants à l'anémie n'est pas soutenue par des preuves scientifiques. En effet, de petites études ont montré que les gens âgés tolèrent l'hémodilution à un hématocrite de moins de $30 \%{ }^{13}$ De plus, les résultats de l'étude TRICC n'ont pas montré de différence de mortalité chez les patients de plus de $\mathbf{5 5}$ ans transfusés à une concentration d'hémoglobine de $70 \mathrm{~g} \cdot \mathrm{L}^{-1}$ comparée à une concentration de moins de $100 \mathrm{~g} \cdot \mathrm{L}^{-1}{ }^{7}$ Mais il est bien prouvé que l'âge est un facteur de risque pour la transfusion. ${ }^{14}$ Il faut répondre à la question de savoir si l'âge modifie les seuils d'hémoglobine, car avec une population vieillissante, les transfusions fondées sur l'âge peuvent influencer l'utilisation de culots globulaires.

Le chemin qui mène à une pratique transfusionnelle optimale compte certaines étapes. En plus de tenter de remplacer la transfusion et de rechercher des marqueurs physiologiques d'hypo-oxygénation tissulaire, nous devons mieux comprendre quels facteurs influencent la prise de décision clinique en pratique transfusionnelle, s'il y a des facteurs reliés au patient, aux politiques institutionnelles et/ou une meilleure preuve à partir des publications approuvées par les pairs. À cette fin, Turgeon et coll. nous apportent de nouvelles informations. Les prochaines étapes seront de déterminer si la transfusion fondée sur les facteurs reconnus dans cette étude a un effet sur la morbidité et la mortalité du patient.

\section{References}

1 Spiess BD. Risks of transfusion: outcome focus. Transfusion 2004; 44: 4S-14S.

2 Hebert PC, Qun Hu L, Biro GP. Review of physiologic mechanisms in response to anemia. CMAJ 1997; 156(suppl): S27-S40.

3 Kleinman S, Chan P, Robillard P. Risks associated with transfusion of cellular blood components in Canada. Transfus Med Rev 2003; 17: 120-62.

4 Corwin HL, Gettinger A, Pearl RG, et al. The CRIT Study: Anemia and blood transfusion in the critically ill-current clinical practice in the United States. Crit Care Med 2004; 32: 39-52.

5 Weiskopf $R B$. Do we know when to transfuse red cells to treat acute anemia? Transfusion 1998; 38: 517-21.

6 Turgeon AF, Fergusson DA, Doucette S, Khanna MP, Tinmouth A, Hebert PC. Red blood cell transfusion practices amongst anesthesiologists: a survey. Can J Anesth 2006; 53: 344-52.

7 Hebert PC, Wells G, Blajchman MA, et al. A multicenter, randomized, controlled clinical trial of transfusion requirements in critical care. Transfusion Requirements in Critical Care Investigators, Canadian Critical Care Trials Group. N Engl J Med 1999; 340: 409-17.

8 Nuttall GA, Stehling LC, Beighley CM, Faust RJ; American Society of Anesthesiologists Committee on Transfusion Medicine. Current transfusion practices of members of the American Society of Anesthesiologists: a survey. Anesthesiology 2003; 99: 1433-43.

9 American Society of Anesthesiologists Task Force on Perioperative Blood Transfusion and Adjuvant Therapies. Practice guidelines for perioperative blood transfusion and adjuvant therapies: an updated report (October 2005). Available from URL; http://www. asahq.org/publicationsAndServices/BCTGuidesFinal. pdf.

10 Stehling LC, Ellison N, Faust RJ, Grotta AW, Moyers $J R$. A survey of transfusion practices among anesthesiologists. Vox Sang 1987; 52: 60-2.

11 Wu WC, Rathore SS, Wang $\Upsilon$, Radford MJ, Krumbolz $H M$. Blood transfusion in elderly patients with acute myocardial infarction. N Engl J Med 2001; 345: 1230-6.

12 Rao SV, Jollis JG, Harrington RA, et al. Relationship of blood transfusion and clinical outcomes in patients with acute coronary syndromes. JAMA 2004; 292: 1555-62.

13 Vara-Thorbeck R, Guerrero-Fernandez Marcote JA. Hemodynamic response of elderly patients undergoing major surgery under moderate normovolemic hemodilution. Eur Surg Res 1985; 17: 372-6.

14 Covin R, O'Brien M, Grunwald G, et al. Factors affecting transfusion of fresh frozen plasma, platelets, and red blood cells during elective coronary artery bypass graft surgery. Arch Pathol Lab Med 2003; 127: 41523. 